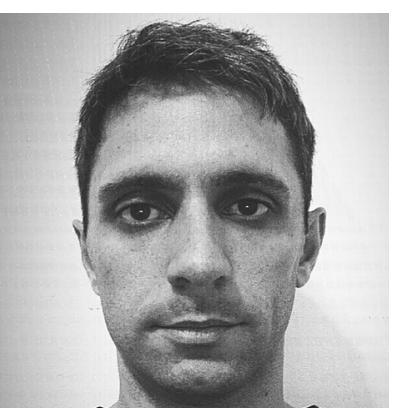
Andrés Rabosto
Lic. en Sociología
Equipo e-TCS, Centro CTS,
Universidad Maimonides y CONICET
andres.rabosto@gmail.com

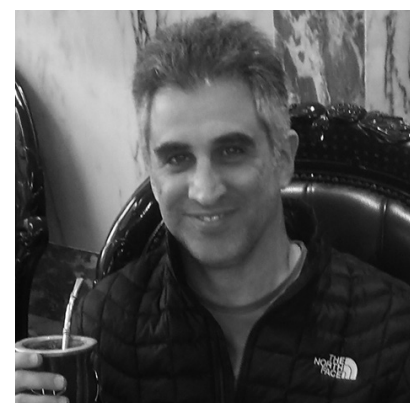

Mariano Zukerfeld

\title{
El sector argentino de software: desacoples entre empleo, salarios y educación
}

Resumen: En las últimas dos décadas el sector de software y servicios de informáticos (SSI) argentino ha experimentado un crecimiento notable. No obstante, los actores empresariales y académicos han señalado de manera reiterada que la escasez de recursos humanos calificados (graduados en carreras afines a la informática) constituye el principal obstáculo a una mayor expansión del sector. Continuando con trabajos previos, aquí se muestra que la evidencia empírica no respalda este argumento y que los datos permiten concluir en cambio, de manera cuantitativa, que el sector SSI no parece dispuesto a compensar económicamente a los profesionales por tener el título universitario, de manera de hacer atractiva su inserción en el sector. Complementariamente, se aportan elementos para comprender por qué el crecimiento del sector no está asociado a un incremento en el número de graduados, sino que, por el contrario, se da en un contexto de escasez de profesionales con título universitario. Finalmente se proponen algunos lineamientos generales para la elaboración de una nueva legislación que promueva y regule la actividad del sector.

Palabras clave: Sector software y servicios informáticos, salarios, educación, empleo, políticas públicas, sindicalismo, trabajo y tecnologías digitales

The Argentinean software sector: Mismatches between employment, wages and education.

Abstact: In the last two decades, the Argentinean Software and Information Services sector (SIS) has experienced a remarkable growth. However, businessmen and scholars have repeatedly pointed out that the shortage of qualified human resources (ie. graduates in computer science related careers) constituted the main obstacle to further expansion of the sector. Continuing with previous works, here it is shown that the empirical evidence does not support this argument and that the data allow to conclude instead, in a quantitative way, that the SSI sector does not seem willing to compensate professionals economically for having the university degree, in a way to make its insertion in the sector attractive. In addition, elements are provided to understand why the growth of the sector is not associated with an increase in the number of graduates, but, on the contrary, occurs in a context of shortage of professionals with a university degree. Finally some general guidelines are proposed for the elaboration of a new legislation that promotes and regulates the activity of the sector.

Keywords: Software and Information Services sector, employment, education, wages, public policies, unions, informational work 
| Ciencia, Tecnología y Política | Año 2 | No22 | Enero-Junio 2019 | ISSN 2618-3188 | www.revistas.unlp.edu.ar/CTyP |

\section{O setor de software argentino: Dissociação entre emprego, salários e educação}

Resumo: Nas últimas duas décadas, o setor argentino de software e serviços de informação (SSI) experimentou um crescimento notável. No entanto, os atores empresariais e acadêmicos têm repetidamente assinalado que a escassez de recursos humanos qualificados (graduados em carreiras relacionadas à ciência da computação) constitui o principal obstáculo para uma maior expansão do setor. Dando continuidade a trabalhos anteriores, mostraremos aqui que a evidência empírica não suporta esse argumento e que os dados permitem concluir em vez disso que, quantitativamente, o setor de SSI não parece disposto a compensar financeiramente os profissionais para ter um diploma universitário, de modo a fazer atrativa sua inserção no setor. Além disso, os elementos são fornecidos para entender por que o crescimento do setor não está associado a um aumento no número de graduados, mas sim a um contexto de escassez de profissionais com diploma universitário. Finalmente, algumas diretrizes gerais são propostas para a elaboração de uma nova legislação que promova e regule a atividade do setor.

Palavras-chave: setor de software e serviços de informática - salários - educação - emprego - políticas públicas - sindicalismo - trabalho e tecnologias digitais

\section{Introducción}

El sector de software y servicios de informáticos (SSI) es, cada vez más, clave en los sistemas nacionales de innovación. De hecho, se trata del sector que provee el principal medio de producción de la actual etapa del capitalismo, ya que, el software, "encarna la función de producción de la economía en sí misma, proporcionando una plataforma para la innovación en todos los sectores" (Jorgenson, 2006:5). Desde comienzos del siglo XXI, se espera que los países en desarrollo se centren en el SSI (UNCTAD, 2012) y en nuestro país existe un consenso sobre las potencialidades del SSI para aumentar las exportaciones intensivas en conocimiento, el empleo calificado y, en general, lograr el catch up para integrarse a la llamada "economía de servicios basados en conocimiento" (López, 2018)

En Argentina la producción de software para el mercado comenzó en la década de 1970. Durante tres décadas, el sector creció moderadamente, en un entorno de ausencia de políticas públicas específicas para el mismo. El primer despegue se registró en la década de los noventa, junto con la expansión del mercado de computadoras personales e Internet. Sin embargo, el punto de inflexión lo marcó la devaluación de 2002, que permitió una primera ola de exportaciones y abarató considerablemente el costo laboral. Desde entonces, las ventas, las exportaciones y el empleo en este campo crecieron a tasas elevadas.

En este contexto de auge, la expansión fue acompañada por la "ley de promoción de la industria de software" (LPS), promulgada en 2004 ${ }^{1}$. La vigencia de la LPS concluye en diciembre de 2019 y sería reemplazada

\footnotetext{
1'De acuerdo al especialista del sector Gabriel Baum, la LPS se trató "de una ley de promoción de la oferta con orientación a la exportación y se apoyó en una estrategia subyacente de crecimiento del sector en sí mismo" (comunicación personal con Gabriel Baum, 04/02/2019). Por ello, de acuerdo a Baum, las metas estuvieron concentradas en la búsqueda de negocios en el exterior antes que en la integración de cadenas de valor con sectores locales o en el desarrollo de un mercado local diversificado. Sobre el impacto de la LPS en las exportaciones Vease FIEL (2018); Castro y Jorrat (2013).
} 
por una legislación más amplia acerca de "servicios basados en el conocimiento"2, en el marco de señales políticas poco alentadoras en esta área ${ }^{3}$.

Ciertamente, el SSI ha mostrado una expansión vertiginosa: entre 2004 y 2017 la facturación creció un 343\% mientras que las exportaciones lo hicieron un 773\%, representando en 2017 un 45\% de las ventas del sector y un $0,46 \%$ del comercio mundial de servicios informáticos. Frente a los déficit de balanza comercial sistemáticos de la economía nacional, el SSI mostró en todo el período una balanza comercial positiva (FIEL, 2018). Sin embargo, uno de los puntos más notables en la expansión del sector es su dinámica de creación de empleo registrado: ha crecido un 300\% entre 2002 y 2017, siendo el ámbito de mayor crecimiento relativo del empleo desde la salida de la convertibilidad (Rabosto y Zukerfeld, 2017).

Ahora bien, los actores empresariales y académicos han señalado reiteradamente que la escasez de recursos humanos calificados (entendidos como graduados en carreras afines a la informática) constituía el principal obstáculo para una mayor expansión de este sector (vease por ejemplo Cámara Argentina de la Industria del Sofware -CESSI-, 2018, FIEL, 2018). Sin embargo, esta idea ha sido debatida en trabajos previos (Rabosto y Zukerfeld, 2017; Zukerfeld, 2014; Ferpozzi y Zukerfeld, 2012; Dughera et al, 2012). En este artículo se presenta nueva evidencia cuantitativa que demuestra las limitaciones y, más aún, el carácter ideológico de tal postulado.

\section{Evolución del empleo, estudiantes y graduados en el sector SSI}

Es incuestionable que, desde el salto expansivo del SSI, los puestos de trabajo fueron cubiertos cada vez más por empleados sin título universitario.

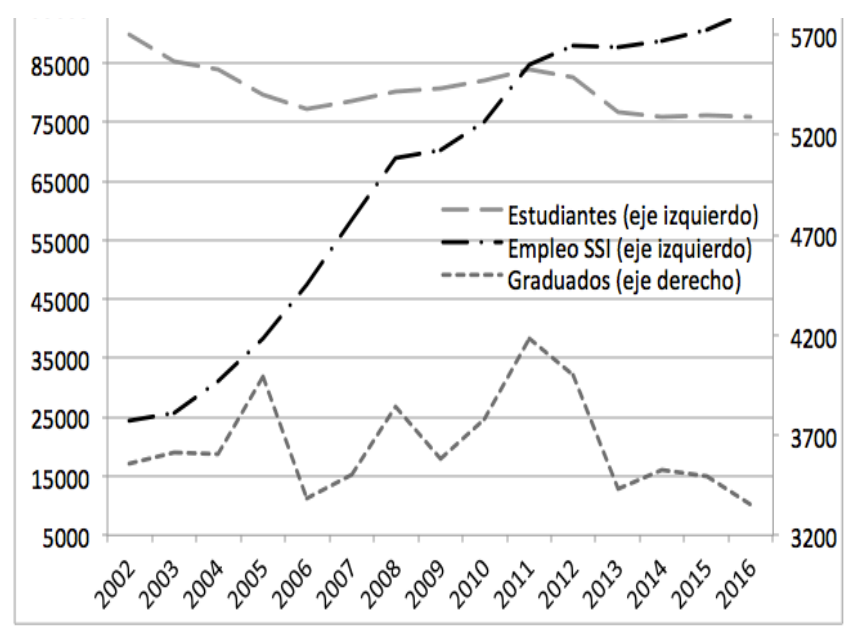

Gráfico 1. Evolución del empleo en el sector SSI, de estudiantes y graduados de carreras informáticas de todo el país (2001-2017). Fuente: elaboración propia en base a SPU y OEDE

Hasta fines de los 90, las curvas de evolución de la cantidad de estudiantes de carreras informáticas de todo el país y las del empleo en el sector SSI tenían pendientes paralelas (Zukerfeld, 2014). Sin embargo, como se puede apreciar en el gráfico 1, desde el despegue del sector, se verifica un crecimiento exponencial del empleo que es al menos parcialmente independiente de los estudiantes activos y egresados de carreras informáticas.

\footnotetext{
${ }^{2}$ Actualmente el ministerio de producción trabaja en una nueva ley para promover los "servicios basados en el conocimiento". Esta ley reemplazaría y absorbería a la LPS.

${ }^{3}$ El presupuesto 2019 grava a las exportaciones de servicios incluyendo a los informáticos (que hasta el momento se encontraban exentos) y fija una carga tributaria del $12 \%$ para las mismas.
} 
Si bien el cruce entre ambas curvas se explica por el crecimiento exponencial del empleo, no deja de ser llamativo que la cantidad absoluta de estudiantes activos tenga una leve caída, pese a los esfuerzos en expandir la oferta y la matrícula de estas carreras. Por otra parte, como se muestra en el gráfico 2, si observamos la proporción de graduados informáticos en relación al total de estudiantes de carreras informáticas, vemos que se encuentra estancada desde el 2004 en torno al 4,5\%. Y como proporción de los graduados de todas las carreras, se encuentra en caída pasando de un 4,5\% en 2004 a un 2,7\% en 2016. Al mismo tiempo, la tasa de graduados de carreras informáticas es sistemáticamente menor a la tasa de graduados del total de carreras.

Por tanto, si por recursos humanos calificados se entiende estudiantes activos y/o graduados de educación superior en informática, la escasez parece ser incuestionable.

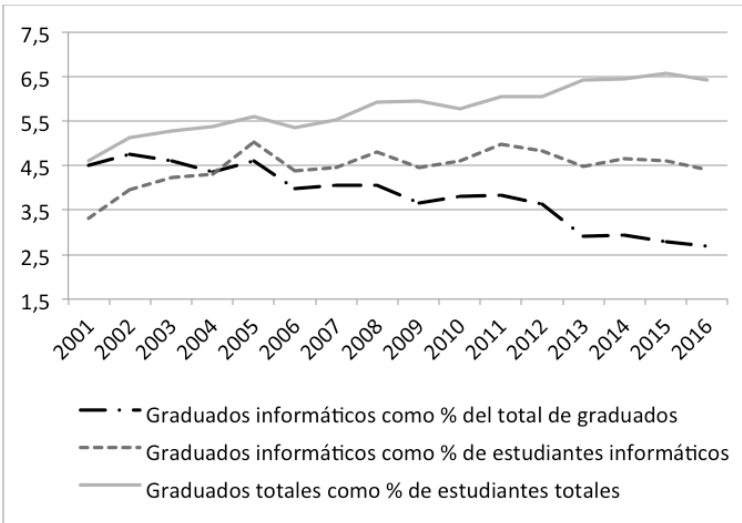

Gráfico 2. Evolución de la tasas de graduados en informática Fuente: elaboración propia en base a SPU

En tal situación, ante una demanda creciente y una oferta estancada, en los términos económicos neoclásicos, sería esperable que: I- las titulaciones académicas para los trabajadores del sector implicaran rendimientos salariales elevados y que II- los salarios hubieran tenido una pendiente creciente. A continuación, se examinan ambas hipótesis.

\section{Tener un título no incrementa el salario en el sector SSI}

¿Qué ha sucedido con los rendimientos salariales de los títulos universitarios y los estudios de posgrado y, más en general, con el rendimiento salarial aportado por el incremento en cada año de escolaridad formal? Sorpresivamente, el sector SSI muestra los rendimientos salariales más bajos del conjunto de sectores laborales del país para cada escalón de logro educativo, particularmente en lo que refiere a los títulos de grado y estudios de posgrado (Rabosto, 2018). Mientras que, para el conjunto de los sectores laborales (agregado), tener un título universitario incrementa en promedio un 50\% el salario horario, en el sector de SSI lo hace solo en un $18 \%$. Por otra parte, mientras que en el agregado poseer estudios de posgrado incrementa en promedio un $12 \%$ el salario horario frente a quienes tienen estudios universitarios completos, en el sector de software lo hace sólo un 1\%. Asimismo, la estimación del rendimiento salarial aportado por cada año de escolaridad formal indica que el mismo es en promedio de 6,3\% para el sector de software frente a un 9,6\% en el conjunto de los sectores laborales. Podría pensarse que las bajas tasas de retorno son una característica de la industria del software global. Sin embargo, en los EEUU los rendimientos del sector de software se encuentran entre los más altos de la economía: los títulos de grado otorgan en promedio un $60 \%$ de incre- 

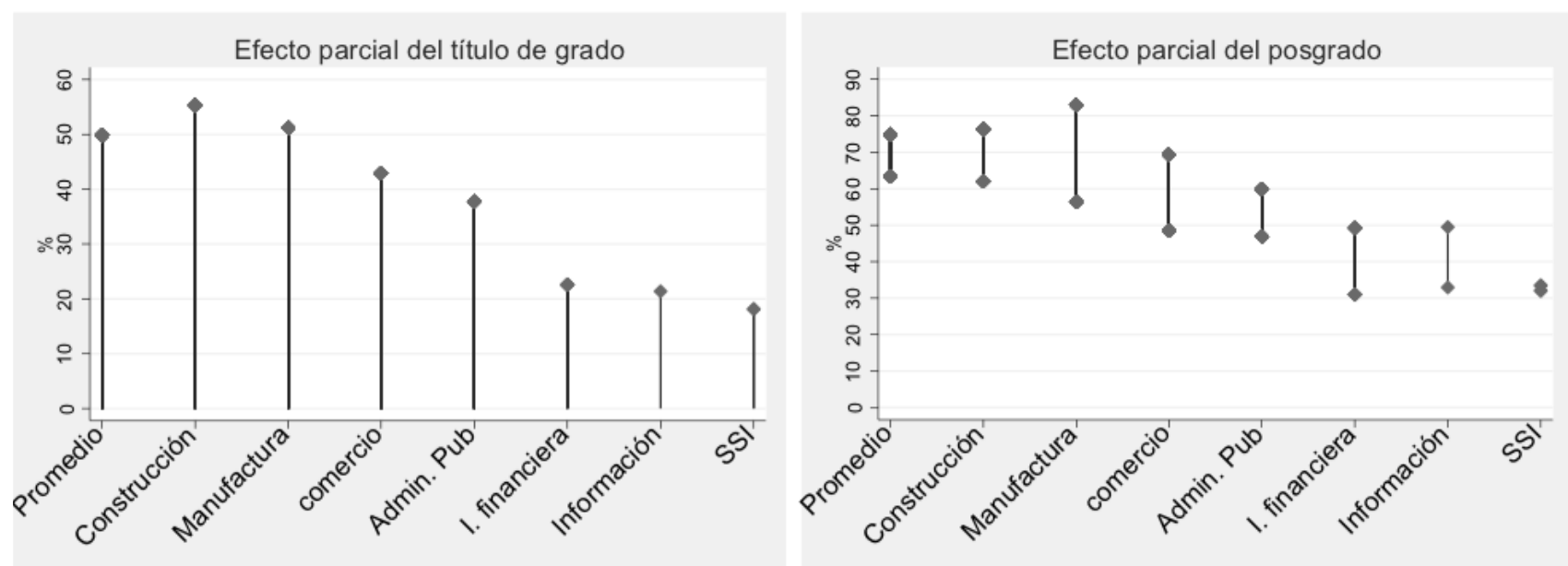

Gráfico 3. Rendimiento promedio del título de grado y los estudios de posgrado sobre el salario horario por sector. Fuente: elaboración propia en base a EPH (pool 2011-2017)

mento en el salario, frente a un 45\% para el promedio del resto de los sectores, mientras que los retornos por años de escolaridad son de un 15\% para la industria de software frente a un 9\% del agregado (Rabosto, 2018). El gráfico 3 muestra en nuestro país los rendimientos salariales de los títulos de grado y lo estudios de posgrado, respectivamente.

Finalmente, para un cuadro más completo, es interesante cruzar el enfoque sectorial con el ocupacional, es decir, comparar lo que ocurre con los empleados que trabajan en ocupaciones de software dentro del SSI respecto de quiénes ejercen tales ocupaciones en otros sectores (por ejemplo, un desarrollador que trabaja en el sector financiero).

Al hacerlo, se observa que aquellos empleados en ocupaciones de software que poseen título universitario tienen más probabilidad de ser contratados por sectores ajenos al SSI que por éste (Rabosto, 2018). De modo que el sector de SSI tiende a contratar sólo a un número limitado de los graduados disponibles.

¿Por qué aquellos empleados en ocupaciones de software que lograron obtener un título no optan por desarrollarse dentro del sector de SSI que, al parecer, los reclama? El salario parece ser un elemento clave: los ingresos de quienes trabajan en ocupaciones de software son más elevados fuera del sector SSI, sobre todo si son graduados, que dentro de él (Zukerfeld y Rabosto, 2017). En suma, las empresas del sector SSI no parecen dispuestas a traducir su declamado interés por más trabajadores con título, en salarios suficientemente atractivos.

\section{Salarios y sindicalización en el sector SSI}

En lo que refiere a la segunda hipótesis, si bien es cierto que los salarios en el sector de SSI se mantuvieron siempre por encima del promedio del sector privado registrado, un análisis de la evolución de los mismos muestra que desde la salida de la convertibilidad ha tenido los incrementos nominales más bajos de toda la economía y, peor aún, si se ajustan por inflación, es el único sector donde el poder adquisitivo del salario promedio ha caído entre 1998 y 2015. Según nuestras estimaciones la caída fue del 20\% (Rabosto y Zukerfeld, 2017). Así, frente a los supuestos neoclásicos, el sector de SSI nos pone ante la extraña paradoja de mostrar el crecimiento más dinámico del empleo de todo el sector privado, junto a los menores incrementos salariales, al tiempo que sus voceros señalan como barrera la disponibilidad de trabajadores calificados. 
| Ciencia, Tecnología y Política | Año 2 | No22 | Enero-Junio 2019 | ISSN 2618-3188 | www.revistas.unlp.edu.ar/CTyP |

Por supuesto, no es sorprendente encontrar que la realidad y la teoría económica neoclásica no coinciden. Sin embargo, ¿cuál podría ser la causa de una divergencia tan significativa? La explicación debe buscarse, creemos, no en aspectos técnicos de la economía sino en la dinámica política y sindical: la ausencia de reconocimiento oficial a los sindicatos específicos del sector es un factor clave. Junto a esto, la baja tasa de afiliación también es destacable, además de la falta de representación colectiva4.

La ausencia de sindicatos con personería gremial privó a los trabajadores de una representación colectiva que pueda negociar un convenio colectivo y regular las condiciones de trabajo, al tiempo que favoreció la negociación individual. Las empresas aprovecharon esto negociando con sindicatos ajenos a la actividad, que ofrecen acuerdos desfavorables para los trabajadores. La sindicalización en organizaciones destinadas a otras actividades económicas contribuye a generar una situación de distanciamiento y ajenidad tal, que los trabajadores optan por negociar por fuera del marco sindical. Ambos factores favorecen el salario personalizado, los bonos y penalidades de productividad, la individualización y diferenciación (Rabosto y Zukerfeld, 2017). Sin embargo, a fines de 2018 la Asociación Gremial de Computación ha obtenido la personería gremial ${ }^{5}$, por lo que podrían producirse modificaciones en este estado de cosas.

De este modo, los datos expuestos en las secciones anteriores nos permiten señalar que: I- el crecimiento del empleo fue parcialmente independiente de la oferta de estudiantes activos y graduados informáticos;

II- esto no parece haber limitado la expansión del empleo en el SSI, que muestra la tasa de creación de empleo registrado más dinámica del sector privado; III- pese a esto, es el único sector en que cayó el salario real entre la salida de la convertibilidad y 2015; IV- los rendimientos salariales de las titulaciones académicas del sector son las más bajas del sector privado.

\section{Divorcio entre titulación y crecimiento: tres elementos}

¿Cómo se explica que el empleo en el sector SSI haya crecido vertiginosamente en un contexto de escasez de graduados y que, al mismo tiempo, las titulaciones tengan poco impacto en el salario? En una etapa del capitalismo en la que numerosos discursos vinculan la generación y difusión de conocimientos con la creación de riqueza, creemos que, sin embargo, se producen simplificaciones erradas.

Desde mediados de la década del 70, y con más claridad desde los 90, los discursos de organismos multilaterales y sectores académicos sobre la llamada «Sociedad del Conocimiento» (que no es otra cosa que el capitalismo informacional o cognitivo) redujeron los saberes valiosos a aquellos que se transmiten en la universidad y, más grave, asociaron la totalidad de los saberes que circulan en las universidades con aquellos que adquieren quienes obtienen un título. De este modo, se postuló la existencia de un vínculo lineal entre titulaciones académicas y crecimiento económico. Sin embargo, ese vínculo es complejo y no se verifica de modo causal ni inmediato.

Si bien los saberes validados por la titulación académica siguen siendo importantes en diversas discipli-

${ }^{4} 8$ La tasa de afiliación sindical en el sector privado registrado es de alrededor del 37\%, mientras que el 39\% de los trabajadores tiene un representante sindical en su lugar de trabajo (Novick, 2012: 14). Sin embargo, en el caso de SSI, nuestras estimaciones indican que existe un máximo del 8\% de la fuerza de trabajo afiliada a una organización sindical o asociación profesional específica del sector (Rabosto y Zukerfeld, 2017).

${ }^{5}$ Para agremiar a los trabajadores que realicen tareas de "operador, programador, analista, soporte técnico, desarrollo de sistemas, centro de cómputos, técnicos" con zona de actuación en CABA y la Provincia de Buenos Aires. (Res. 239-2018 del APN-SGTYE-MPYT, 29/11/2018). Sin embargo, a mediados de marzo de 2019 la resolución no se ha publicado aun en el Boletín Oficial y numerosas firmas del sector se basan en este hecho para no avanzar en la discusión de un convenio colectivo de trabajo, según señala Ezequiel Tosco, Secretario General de la AGC. 
nas en relación a la creación de riqueza, a nivel agregado y especialmente en sectores característicos del capitalismo informacional o cognitivo (software, contenidos audiovisuales, etc.) hay cierto desacople entre las titulaciones y los saberes que impactan en la productividad. Esto es especialmente cierto para el sector SSI (Zukerfeld, 2014). ¿Por qué se produce este divorcio entre el crecimiento de la productividad global del sector y el de las titulaciones académicas? Aunque este tema merece una discusión mayor, señalamos al menos tres razones:

En primer lugar, por la existencia de saberes impartidos para obtener un título universitario que tienen poco o nulo impacto en el sistema productivo y en la sociedad en general. Una porción no desdeñable de los saberes que se adquieren en la universidad puede resultar obsoleta respecto de procesos productivos que mutan a una velocidad considerable. El hecho de que las reformas de los programas de estudios deban ser aprobadas por numerosas instancias burocráticas, junto con los tiempos necesarios para la capacitación estandarizada y masiva de los docentes, limita la adaptabilidad de algunas instituciones de educación superior. Por supuesto, no se trata aquí de apoyar la agenda neoliberal consistente en destruir los resguardos del sistema de educación pública contra los caprichos del mercado, sino más bien de lo contrario: notar la obsolescencia para que la adaptación se haga en función de las necesidades de la sociedad que financia el sistema educativo, que incluye pero excede largamente al ámbito mercantil, y cuyas necesidades en muchos casos se contraponen directamente con las de aquél.

En segundo lugar, el sector SSI ha crecido en base a saberes productivos que se adquieren en la universidad sin requerir un título -entre los mal llamados "desertores". En efecto, los estudiantes que pasan por la universidad adquieren saberes relevantes no sólo para ellos, sino para la sociedad e incluso para el desarrollo económico, se gradúen o no. Notablemente, la mayor parte de las figuras más reconocidas por quiénes pregonan la importancia de la titulación en el camino hacia el desarrollo pasaron por la universidad, pero no se graduaron. ${ }^{\circ} ¿$ Quiere decir esto que no tuvo sentido que fueran a la universidad? No, en modo alguno. Puede comprobarse que los estudiantes que no obtienen un título adquieren numerosos saberes gracias a su paso por la educación superior formal. Entre ellos: i. saberes específicos de determinadas asignaturas, conocimientos técnicos útiles. ii. Redes de reconocimiento (o capital social), es decir, de "contactos" o conocidos (weak ties) que pueden ser cruciales en el inicio de emprendimientos, o la obtención de empleos: colegas, socios, profesores, inversores o empleadores. iii. Pautas organizacionales y axiología: el acostumbramiento a determinadas reglas relativas a la administración de recursos, planificación, cumplimiento de reglas y compromisos y, especialmente, la adecuación de medios a fines -es decir, la racionalidad instrumental- resulta un beneficio extremadamente relevante de la dinámica universitaria.

En tercer lugar, saberes productivos que se adquieren por fuera de la universidad. Esta es quizás la principal explicación del crecimiento el sector SSI (aunque se trata de una fuerza que lo excede e involucra a la transmisión de conocimientos en el capitalismo informacional). Se vincula con la adquisición de saberes antes asociados a la universidad, pero ahora por fuera de ella y con modalidades ajenas a su lógica. Se trata de saberes individuales (típica pero no exclusivamente informacionales), pero también de destrezas intersubjetivas. Naturalmente, nos referimos aquí a modalidades vinculadas con Internet y las tecnologías digitales, como el autoaprendizaje, la utilización de tutoriales, el aprendizaje entre pares, las comunidades

${ }^{6}$ Algunos ejemplos incluyen a figuras como Michael Dell (fundador de Dell), Steve Jobs (fundador de Apple), JulianAssange (fundador de Wikileaks), Bill Gates (fundador de Microsoft), Evan Williams (co-fundador de Twitter), Mark Zuckerberg (fundador de Facebook), Larry Ellison (fundador de Oracle), Jan Koum (fundador de WhatsApp) y Travis Kalanick (fundador de Uber). 
de prácticas, el aprendizaje basado en juegos y otras.

\section{A modo de cierre: lineamientos de política pública}

En el contexto de las discusiones respecto de una nueva legislación que promueva y regule la actividad del sector proponemos algunos lineamientos generales que se basan en el presente trabajo -y tangencialmente en anteriores-. Se trata de aportes para la discusión y no de recetas consolidadas.

Sobre la relación entre Economía y Trabajo: Las legislaciones que ofrezcan beneficios para firmas del sector podrían ofrecer incentivos específicos para aquellas que provean condiciones de trabajo (incluyendo aspectos salariales y sindicales) más favorables y eslabonamientos productivos más fértiles con otros sectores. La legislación podría impulsar y contemplar la sindicalización de los trabajadores en gremios específicos que permita que tanto los aspectos salariales como las condiciones y el ambiente de trabajo sean discutidos por representantes que desarrollen esta actividad y, más importante, que permita concretar un convenio colectivo de trabajo adecuado a la misma. Ambas cuestiones se vinculan con un precepto más profundo: la política para el sector debe dejar de basarse en asunciones de la economía neoclásica (como que la demanda de trabajo generará alza salarial en el sector, o que el bono salarial por titulación genera automáticamente un incentivo significativo para los trabajadores) que, al menos en este sector, no se verifican.

Sobre la formación de recursos humanos: Las políticas para este sector deberían contemplar las funciones que tienen tanto la educación formal (con o sin titulación), como la no formal e informal, de manera sistémica. En ese sentido resulta crucial indagar las necesidades específicas del sistema productivo argentino como totalidad, que exceden a la búsqueda de beneficios de una porción del sector empresarial. La formación para procesos productivos de software en el Estado (nacional, local, universidades y otros organismos, etc), en cooperativas, en comunidades de software libre y en la producción in-house puede tener prioridades distintas que las de las empresas, pero no menos importantes para una estrategia de desarrollo. Así, las políticas públicas deberían evitar dos riesgos: la definición de una agenda cognitiva orientada sólo en términos de producción de mercancías (incluyendo subjetividades mercantiles) y del alineamiento con las necesidades de procesos productivos exógenos.

Creación de un observatorio estadístico para el SSI: Este trabajo intenta hacer aportes basados en evidencias empíricas. Sin embargo, los datos con los que se cuenta son muy limitados. Tanto los rasgos del sector (p.ej. presencia de numerosas microempresas que sin embargo no necesariamente tienen baja productividad) como de los instrumentos de medida (p.ej. la Encuesta Permanente de Hogares (EPH) presenta dificultades estadísticas para capturar el empleo en el sector adecuadamente) limitan el diagnóstico y dificultan la calibración de las políticas públicas. Los valiosos esfuerzos realizados por el Observatorio Permanente de la Industria de Software y Servicios Informáticos (OPSSI) de la Cámara de la Industria Argentina del Sofware (CESSI) han tenido la entendible limitación de que producen mediciones útiles para un subconjunto del sector empresarial. En cambio, parece necesaria la creación de un observatorio estadístico del sector con financiamiento estatal y autonomía que permita tanto sistematizar la información secundaria como producir fuentes primarias. 


\section{Bibliografía}

Castro, L., y Jorrat, D. (2013). "Evaluación de impacto de programas públicos de financiamiento sobre la innovación y la productividad: el caso de los servicios de software e informáticos de la Argentina”. Documento de trabajo No. 2013 (SS-IP)-06.

CESSI - REPC (2018). "Plan Estratégico Federal de la Industria Argentina del Software 2018-2030", Cessi Argentina - Red de Entidades, Polos y Clústeres, mayo de 2018.

Dughera, L., Segura, A., Yansen, G., \&Zukerfeld, M. (2013). Sobre el aprendizaje de los trabajadores informáticos: Ios roles de la educación formal, no formal e informal en la adquisición de" técnicas". Revista Educación y Pedagogía, 24(62), 79-101.

Ferpozzi, H. y Zukerfeld, M. (2012). ¿Puede el sindicalismo adaptarse al trabajo informacional? En Dughera, L.; Yansen, G.; Zukerfeld, M., (eds.) Gente con códigos. La heterogeneidad de los procesos productivos de software. Buenos Aires: Universidad Maimónides.

FIEL (2018) La economía de la industria argentina del software. La ley de promoción del software y su impacto en la evolución del sector. Comparación internacional. Trabajo para la CESSI.

Jorgenson, Dale (2006) "Introduction: Software and the New Economy" en Jorgenson, dale w. and Wesener Charles W., (Editors) (2006) Measuring and Sustaining the New Economy, Software, Growth, and the Future of the U.S Economy. National Research Council.

López, A. (2018). Los servicios basados en conocimiento ¿una oportunidad para la transformación productiva en Argentina? Buenos Aires: Serie Documentos de Trabajo del IIEP FCE UBA.

Rabosto, A. (2018). Dimensiones del salario en el trabajo Informacional. Una evaluación del efecto parcial de la educación formal. Workingpaper. Presentado en seminario CCTS, B.S.A.S. 18/12/2018

Rabosto, A., yZukerfeld, M. (2017). Precarity, precariousness and software workers: wages, unions and subjectivity in the Argentinian software and information services sector. WorkOrganisationLabour\&Globalisation, 11(1), 87-102

Zukerfeld, M y Rabosto, A (2017) Producción de Software y Sector SSI: una mirada sobre los salarios y la educación. Presentación en la UNCPBA, Tandil, 29/11/2017.

Zukerfeld, M. (2014).Revisiting the mismatch between formal education in computer science and the software and information services sector: the case of Argentina. Prometheus, 32(2), 181-201 